\title{
Molecular Neuropsychology: Creation of Test-Specific Blood Biomarker Algorithms
}

\author{
Sid E. O'Bryant ${ }^{a} \quad$ Guanghua Xiao $^{d}$ Robert Barber ${ }^{c} \quad$ C. Munro Cullum ${ }^{\text {e, }}$ \\ Myron Weiner $^{\mathrm{e}, \mathrm{f}}$ James Hall $^{\mathrm{b}}$ Melissa Edwards $^{\mathrm{h}} \quad$ Paula Grammas $^{\mathrm{i}}$ \\ Kirk Wilhelmsen ${ }^{j}$ Rachelle Doodyk Ramon Diaz-Arrastial \\ Texas Alzheimer's Research and Care Consortium

\begin{abstract}
Departments of a Internal Medicine, ${ }^{\mathrm{b}}$ Psychiatry and ${ }^{\mathrm{C}}$ Pharmacology and Neuroscience, and Institute for Aging and Alzheimer's Disease Research, University of North Texas Health Science Center, Fort Worth, Tex., Departments of ${ }^{\mathrm{d}}$ Clinical Sciences, ${ }^{\mathrm{e}}$ Psychiatry, ${ }^{\mathrm{f}}$ Neurology and Neurotherapeutics and 9 Neurology, University of Texas Southwestern Medical Center, Dallas, Health Sciences Center, Garrison Institute on Aging, Lubbock, Tex., 'j Department of Genetics, University of North Carolina School of Medicine, Chapel Hill, N.C., kDepartment of Neurology, Baylor College of Medicine, Houston, Tex., and 'Center for Neuroscience and Regenerative Medicine, Uniformed Services University of the Health Sciences, Rockville, Md., USA
\end{abstract}

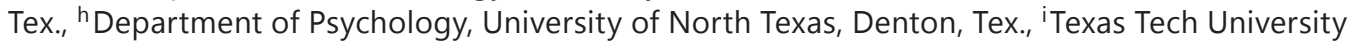

\section{Key Words}

Algorithms $\cdot$ Biomarkers $\cdot$ Molecular markers $\cdot$ Neuropsychology $\cdot$ Psychology

\begin{abstract}
Background: Prior work on the link between blood-based biomarkers and cognitive status has largely been based on dichotomous classifications rather than detailed neuropsychological functioning. The current project was designed to create serum-based biomarker algorithms that predict neuropsychological test performance. Methods: A battery of neuropsychological measures was administered. Random forest analyses were utilized to create neuropsychological test-specific biomarker risk scores in a training set that were entered into linear regression models predicting the respective test scores in the test set. Serum multiplex biomarker data were analyzed on 108 proteins from 395 participants (197 Alzheimer patients and 198 controls) from the Texas Alzheimer's Research and Care Consortium. Results: The biomarker risk scores were significant predictors $(p<0.05)$ of scores on all neuropsychological tests.
\end{abstract}

S.E.O'B. and G.X. contributed equally to this study.

Sid E. O'Bryant, PhD

Department of Internal Medicine, University of North Texas Health Science Center

3500 Camp Bowie Boulevard

Fort Worth, TX 76107 (USA)

E-Mail Sid.O'Bryant@unthsc.edu 
With the exception of premorbid intellectual status (6.6\%), the biomarker risk scores alone accounted for a minimum of $12.9 \%$ of the variance in neuropsychological scores. Biomarker algorithms (biomarker risk scores and demographics) accounted for substantially more variance in scores. Review of the variable importance plots indicated differential patterns of biomarker significance for each test, suggesting the possibility of domain-specific biomarker algorithms. Conclusions: Our findings provide proof of concept for a novel area of scientific discovery, which we term 'molecular neuropsychology'.

Copyright (C) 2013 S. Karger AG, Basel

\section{Introduction}

The long-standing search for accurate biomarkers from many conditions/diseases impacting neuropsychological functioning include, but are not limited to, Alzheimer's disease (AD) [1-4], traumatic brain injury [5, 6], schizophrenia [7, 8], alcohol use/abuse [9], and mood disorders [9-11]. For example, we recently created a serum-based biomarker algorithm that yielded excellent diagnostic accuracy in separating AD cases from controls $[1,12$, 13]. Significant advancements have been made though analyses of blood, cerebrospinal fluid (CSF), and advanced neuroimaging modalities, and it is likely that combining assessment modalities (e.g. biomarkers, clinical data and demographics) will yield better results than any single modality $[1,12,14-16]$.

While animal model work has begun to examine proteomic and genomic methods for discovering potential pathways and biomarkers of specific cognitive abilities [17], the majority of human biomarker research to date has been based on dichotomous group classifications (case vs. control) rather than linear constructs upon which clinical decisions and/or diagnoses of cognitive dysfunction are based (i.e. neuropsychological testing). While others are examining the link between biomarkers of disease states (e.g. AD) and cognitive functioning [18], biomarkers associated with neuropsychological test performance as continuous variables could provide novel opportunities to study these conditions/diseases [4]. For example, in our prior work we did not show a significant difference in serum brainderived neurotrophic factor (BDNF) levels between AD cases and controls; however, serum BDNF levels were specifically associated with memory performance among AD cases [4]. Blood-based biomarkers are preferable as they are more cost and time efficient and more conveniently acquired than CSF or neuroimaging [19]. An additional advantage of proteomic approaches to biomarker identification is the potential to discover alterations at the protein level that may be closely related to the pathophysiological process(es) underlying complex conditions and disease states [9]. The purpose of the present study was to take a first step towards creating serum-based biomarker algorithms of neuropsychological functioning. This proof-of-concept project provides a platform for a novel field of scientific discovery, which we term 'molecular neuropsychology'.

Even though there are currently no available blood-based biomarkers of neuropsychological functioning, there is a growing literature linking specific blood biomarkers to neuropsychological performance. In the instance of AD, we analyzed data from a sample of 399 participants (198 AD cases and 201 controls) enrolled in the Texas Alzheimer's Research and Care Consortium (TARCC) and found that serum BDNF levels were significantly associated with poorer immediate and delayed visual and verbal memory scores [4] among AD cases but not controls. We failed to find a significant link between C-reactive protein (CRP) levels and cognition (Mini-Mental State Examination, MMSE [20]) scores among a sample of 192 AD cases and 174 controls, though increased CRP levels were associated with disease severity [21]. On the other hand, Noble and colleagues [21] analyzed data on 1,331 participants of the 
O'Bryant et al.: Molecular Neuropsychology: Creation of Test-Specific Blood Biomarker Algorithms

WHICAP study and found CRP levels (highest tertile vs. lowest tertile) to be specifically associated with impairment in memory and visuospatial abilities but not language or executive functioning. Additionally, it was noted that apolipoprotein (APO) Eع4 carriers were most likely to demonstrate impairment in memory. Wilson et al. [22] examined a sample of controls, patients with mild cognitive impairment and dementia (AD and non-AD dementia) with clinical dementia rating (CDR) global scores ranging from 0 to 3 . These authors found that RBANS (Repeatable Battery for Neuropsychological Status) [23], language, and immediate and delayed memory indices were significantly related to plasma anti-RAGE IgGs and anti-A $\beta$ IgG concentrations. Interestingly, Ig $\mu$, a heavy chain of IgM, was part of the protein profile recently identified in serum among schizophrenia patients [8] and immunoglobulins were also part of our AD diagnostic algorithm (Appendix 1 of O'Bryant et al. [1]). While neuropsychological testing was not conducted as part of the schizophrenia project, this work suggests a role of immunoglobulins in neuropsychological functioning across multiple disease states. When examining the outcome of children suffering from traumatic brain injury with and without histories of abuse, Beers et al. [24] found serum concentrations of neuron-specific enolase, S100B, and myelin-basic protein were related to 6-month functional and cognitive outcomes. In a sample of 998 non-demented community-dwelling adults of the Longitudinal Aging Study Amsterdam, Dik et al. [25] found that adrenocorticotropic hormone concentration was significantly associated with delayed verbal recall and albumin with MMSE scores. When the sample was restricted to those with MMSE scores $>21$ (suggesting mild-to-moderate dementia), adrenocorticotropic hormone was also associated with processing speed and delayed recall. Next the authors examined only those suspected of possible mild cognitive dysfunction (i.e. MMSE scores 21-26) and found that interleukin (IL)-6 and CRP were significantly associated with cognitive decline over a 3-year period. Despite the growing research linking specific blood-based biomarkers and neuropsychological test performance (by cognitive domain in many cases) across various conditions and disease states, no prior work has attempted to create biomarker algorithms of test-specific neuropsychological functioning.

The current study sought to create neuropsychological test-specific biomarker algorithms in a cohort of elders with and without cognitive impairment. The ability to create such algorithms would have broad-reaching applications. We hypothesized that our test-specific algorithms would account for significant percentages of the variance in neuropsychological performance in the test sample.

\section{Methods}

\section{Participants}

Participants included 395 individuals (197 AD subjects and 198 controls) enrolled in TARCC. The methodology of the TARCC project has been described in detail elsewhere $[1,26]$. Briefly, each participant undergoes a standardized annual examination at the respective sites, which includes a medical evaluation, neuropsychological testing, interview, and blood drawing for storage of samples in the TARCC biobank. Diagnosis of AD was based on NINCDS-ADRDA criteria [27] utilizing consensus review. Controls were subjects without cognitive complaints or medical conditions that could impair cognition and who performed within normal limits on psychometric assessment. Institutional review board approval was obtained at each TARCC site and written informed consent was obtained for all participants.

Assays

Non-fasting blood samples were collected into 10-ml tiger-top serum-separating tubes. Samples were allowed to clot at room temperature for $30 \mathrm{~min}$ in a vertical position. Within $1 \mathrm{~h}$ of collection, samples were centrifuged for $10 \mathrm{~min}$ at 2,500 $\mathrm{g}$ and aliquoted into 1 - $\mathrm{ml}$ cryovial tubes and stored at $-80^{\circ} \mathrm{C}$. Batched spec- 
Dementia

Cognitive Disorders

Table 1. Mean, standard deviation (SD), and least detectable dose (LDD) for top blood-based markers in algorithms

\begin{tabular}{l|l}
\hline \multicolumn{2}{l}{ Dement Geriatr Cogn Disord 2014;37:45-57 } \\
\hline DOI: $\underline{10.1159 / 000345605}$ & $\begin{array}{l}\text { C 2013 S. Karger AG, Basel } \\
\text { www.karger.com/dem }\end{array}$ \\
\hline
\end{tabular}

Algorithms

\begin{tabular}{lrrr}
\hline & Mean & \multicolumn{1}{l}{ SD } & \multicolumn{1}{l}{ LDD } \\
\hline VCAM-1 & 799.79 & 213.14 & $1.5 \mathrm{ng} / \mathrm{ml}$ \\
$\beta_{2}$ M & 2.39 & 1.04 & $0.013 \mu \mathrm{g} / \mathrm{ml}$ \\
IL-8 & 22.95 & 8.87 & $3.5 \mathrm{pg} / \mathrm{ml}$ \\
vWF & 38.26 & 21.32 & $0.40 \mu \mathrm{g} / \mathrm{ml}$ \\
Eotaxin 3 & 104.13 & 333.29 & $70.0 \mathrm{pg} / \mathrm{ml}$ \\
Tenascin C & 957.12 & 639.29 & $3.0 \mathrm{ng} / \mathrm{ml}$ \\
PPY & 192.10 & 191.66 & $5.0 \mathrm{pg} / \mathrm{ml}$ \\
FAS & 8.88 & 4.11 & $0.27 \mathrm{ng} / \mathrm{ml}$ \\
$\alpha_{2}$ M & 1.33 & 1.06 & $0.061 \mathrm{mg} / \mathrm{ml}$ \\
TPO & 6.11 & 1.83 & $3.2 \mathrm{ng} / \mathrm{ml}$ \\
S100B & 0.43 & 0.32 & $0.30 \mathrm{ng} / \mathrm{ml}$ \\
CK-MB & 0.36 & 0.34 & $0.42 \mathrm{ng} / \mathrm{ml}$ \\
SCF & 526.85 & 209.58 & $56.0 \mathrm{pg} / \mathrm{ml}$ \\
EGFR & 4.93 & 1.14 & $0.042 \mathrm{ng} / \mathrm{ml}$ \\
TNF $\alpha$ & 3.50 & 3.61 & $46.0 \mathrm{pg} / \mathrm{ml}$ \\
AgRP & 73.46 & 130.41 & $0.165 \mathrm{ng} / \mathrm{ml}$ \\
G-CSF & 10.06 & 4.84 & $3.4 \mathrm{pg} / \mathrm{ml}$ \\
CRP & 3.45 & 4.52 & $00.0015 \mu \mathrm{mg} / \mathrm{ml}$ \\
\hline
\end{tabular}

VCAM-1 = Vascular cell adhesion molecule $1 ; \beta_{2} \mathrm{M}=\beta_{2}$-microglobulin; IL = interleukin; $\mathrm{vWF}=$ von Willebrand factor; $\mathrm{PPY}=$ pancreatic polypeptide; $\alpha_{2} \mathrm{M}=\alpha_{2}$-macroglobulin; TPO = thyroid peroxidase; $\mathrm{S} 100 \mathrm{~B}=\mathrm{S} 100$ calcium binding protein $\mathrm{B} ; \mathrm{CK}-\mathrm{MB}=$ creatine kinase $\mathrm{MB}$; $\mathrm{SCF}=$ stem cell factor; EGFR = epidermal growth factor receptor; $\mathrm{TNF} \alpha=$ tumor necrosis factor alpha; AgRP = Agouti-related protein; $\mathrm{G}-\mathrm{CSF}=$ granulocyte colony-stimulating factor; $\mathrm{CRP}=\mathrm{C}$-reactive protein

imens from either baseline or year-1 follow-up examinations were sent frozen to Rules Based Medicine (www.rulesbasedmedicine.com; Austin, Tex., USA), where they were thawed for assay without additional freeze-thaw cycles using the Rules Based Medicine multiplexed immunoassay human Multi-Analyte Profile (human MAP). Individual proteins were quantified with immunoassays on colored microspheres. Information regarding the mean, standard deviation, least detectable dose, and interrun coefficient of variation are listed in table 1.

\section{Neuropsychological Testing}

The TARCC neuropsychology core battery consists of commonly utilized instruments that tap a variety of cognitive domains. Specific tests include Digit Span (WAIS-R, WAIS-III, Wechsler memory scale-R, WMS-R) [28], Trail Making Test [29], Logical Memory (LM) and Visual Reproduction (VR; WMS-R and WMS-III) [28], Boston Naming Test (BNT; 30- and 60-item versions) [29], verbal fluency (FAS) [30], Clock Drawing Test [30], AMNART (American National Adult Reading Test) [30], MMSE [20], and CDR [31]. In order to equate scores from digit span and story memory scales, raw scores for all neuropsychological tests were converted to scaled scores based on previously published normative data [32-34]. For the BNT, we recently published an independent study demonstrating the psychometric properties of an estimated 60-item BNT score that can be calculated from 30-item versions [35]; this estimated 60-item score was calculated for all 30-item administrations.

\section{Statistical Analyses}

Analyses were performed using R (V 2.10) statistical software [36]. The log-transformed and then standardized data on each analyte from our AD diagnostic algorithm publication was utilized in the current analyses [1]. Random forest (RF) analysis was developed by Breiman [37, 38] as an ensemble learning method that utilizes a classification tree as the base classifier. The RF model has four steps: (1) generate many 
Table 2. Demographic characteristics of the study sample

\begin{tabular}{l|l}
\hline Dement Geriatr Cogn Disord 2014;37:45-57 \\
\hline DOI: $\underline{10.1159 / 000345605}$ & $\begin{array}{l}\text { @ } 2013 \text { S. Karger AG, Basel } \\
\text { www.karger.com/dem }\end{array}$ \\
\hline
\end{tabular}

O'Bryant et al.: Molecular Neuropsychology: Creation of Test-Specific Blood Biomarker Algorithms

\begin{tabular}{lc}
\hline & Total sample, mean (SD) \\
\hline Gender (male), \% & 33 \\
Age, years & $73.9(9.3)$ \\
Education, years & $14.8(3.2)$ \\
MMSE & $24.4(6.8)$ \\
CDR sum of boxes score & $3.8(5.0)$ \\
APOEc4 carrier, yes/no & $163 / 218(19$ unknown) \\
Hispanic ethnicity, \% & 9 \\
Race, \% & \\
$\quad$ White & 94 \\
$\quad$ Non-White & 6 \\
Diabetes, \% & 11 \\
Hypertension, \% & 60 \\
Hyperlipidemia, \% & 57 \\
Obesity, \% & 17 \\
\end{tabular}

random subsets (bootstrap samples) from the original data; (2) build a decision tree from each random subset; (3) make a prediction from each decision tree model, and (4) combine the predictions from each individual tree model to get the final prediction. The RF model can be used to predict both binary/categorical outcomes and continuous outcomes. For predicting binary or categorical outcomes, the RF model will build many classification trees, and then combine the predictions from individual trees by a majority vote approach. While for predicting continuous outcomes, the RF model will build regression trees [2] instead of classification trees, and then use model-averaging techniques to combine the predictions from individual regression trees. This method has been shown to perform well in many classification and prediction scenarios $[39,40]$, including algorithmic approaches to CSF [41], EEG [42] and fMRI [43, 44] findings. The RF prediction model was performed using R package random Forest (V 4.5) [37], with all software default settings. TARCC participants were randomized to a training set ( $\mathrm{n}=197,98 \mathrm{AD}$ patients and 99 controls) or a testing set $(\mathrm{n}=198$; $99 \mathrm{AD}$ patients and 99 controls) by random number generator. The full list of 108 serum-based analytes utilized in the algorithm can be found in Appendix 1 of O'Bryant et al. [1]. An RF prediction model biomarker risk score was generated for each specific neuropsychological test within the training set, which were then entered as predictor variables in linear regression models in the test set, with the neuropsychological scale scores as the outcome variables; age, gender, and education were entered as covariates. The percentages of participants having a diagnosis of diabetes, hyperlipidemia, hypertension, or being obese are presented in table 2 . With the exception of obesity $(A D=13 \%$, controls $=21 \%)$, there was no significant difference in the presence of these conditions between groups. Obesity status was not significantly related to neuropsychological test scores and, therefore, none of these medical conditions were included in our analyses. Significance was set at $\mathrm{p}<0.05$.

\section{Results and Discussion}

Demographic characteristics of the study population are shown in table 2. The distributions of neuropsychological test scores by AD versus control status can be found in figure 1 . The relation between the most relevant biomarkers and neuropsychological test scores can be found in the heatmap of figure 2. Online supplemental figure 3 provides the distribution of biomarkers by AD versus control status.

The biomarker risk scores created from the training set were significant predictors of all neuropsychological scores in the test set. However, the biomarker risk scores for global cognition (MMSE, $p<0.001$ ) and disease severity (CDR global score $p<0.001$, CDR sum of boxes score $\mathrm{p}<0.001$ ) were among the most powerful. Across the domain of memory, the 


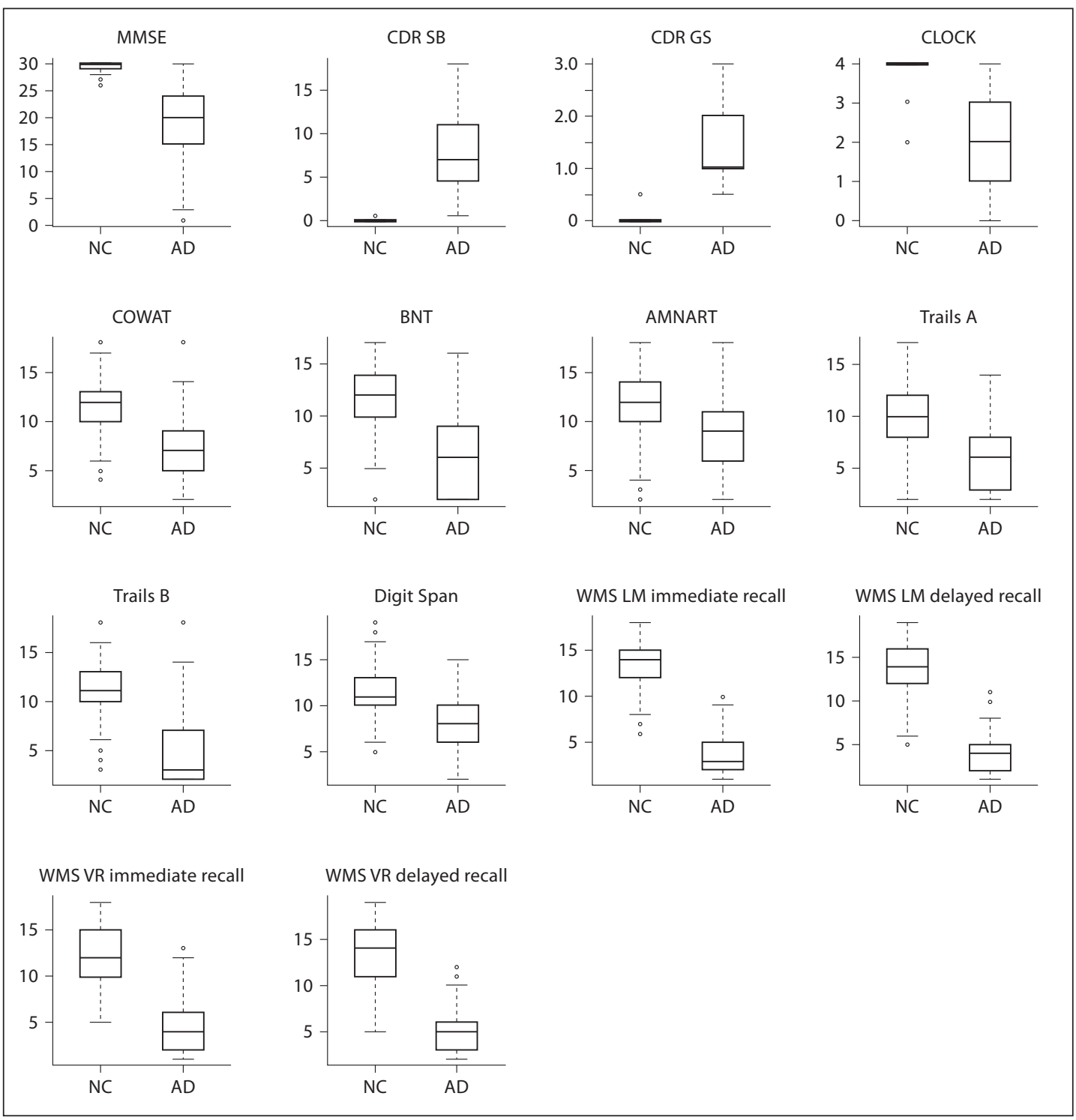

Fig. 1. Distribution of neuropsychological test scores by AD versus normal control (NC).

biomarker risk scores were strong predictors of test performance in both visual (VR I: $\mathrm{p}<$ 0.001 , and VR II: $p<0.001$ ) and verbal domains (LM I: $p<0.001$, and LM II: $p<0.001$; see table 3 for all results).

The amount of variance in test scores accounted for by the biomarker risk scores alone ranged from 6.6 to $47.3 \%$. In fact, the biomarker risk score accounted for $43.9 \%$ of the variance in immediate verbal memory (WMS LM I) and $47.3 \%$ in delayed verbal memory (WMS LM II), $30.9 \%$ of the variance in immediate visual memory (WMS VR I) and $41.2 \%$ of the variance in delayed visual memory (WMS VR II). The amount of variance accounted for by each testspecific biomarker risk score, independent of age, gender and education, is presented in table 3. Prior work has demonstrated that age, gender, and education influence neuropsychological test performance $[29,30]$ and it is standard practice to use these factors in creating normative references. Therefore, we also examined the variance accounted for in neuropsychological 
O'Bryant et al.: Molecular Neuropsychology: Creation of Test-Specific Blood Biomarker Algorithms

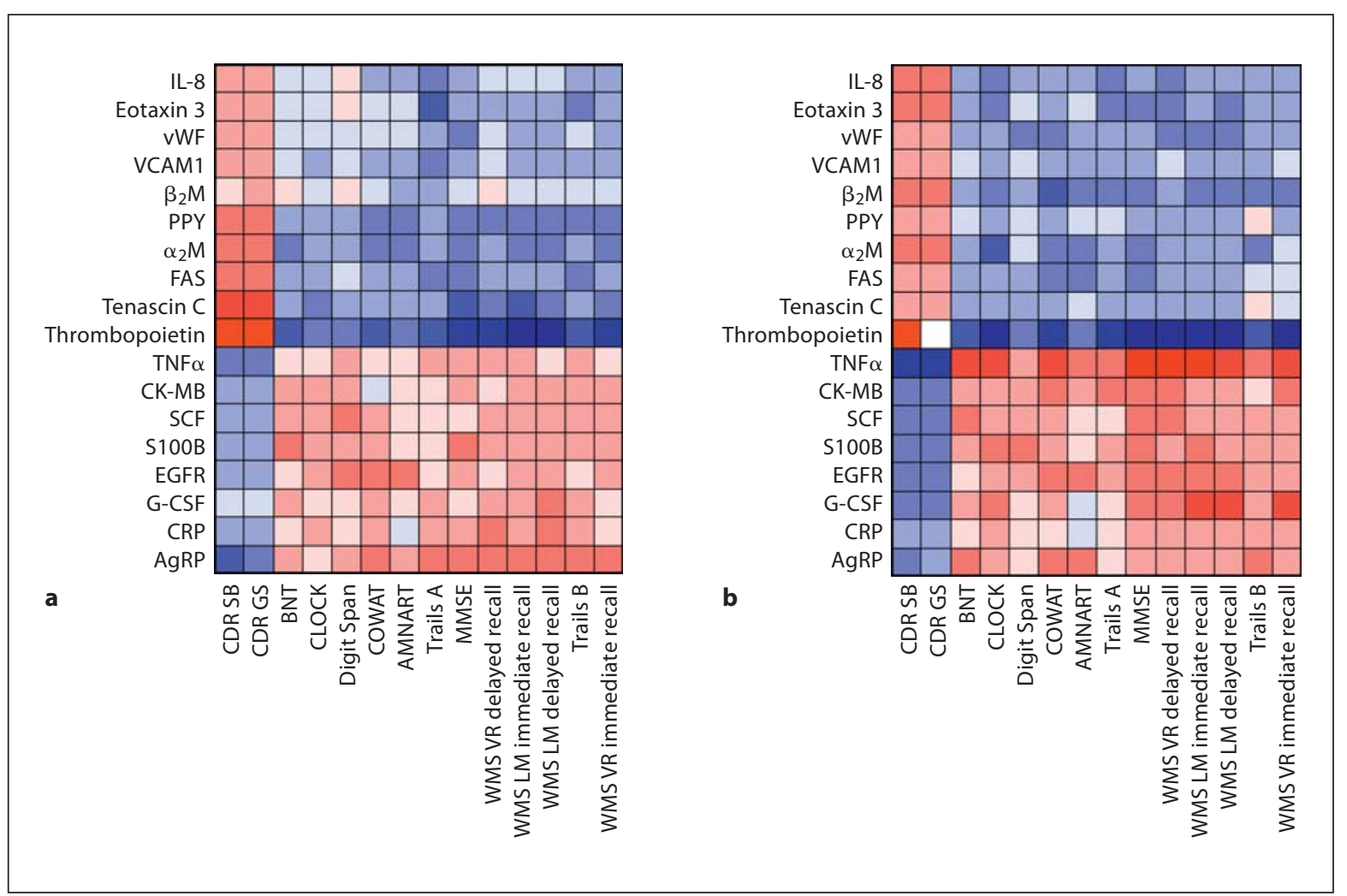

Fig. 2. Heatmap of correlation coefficients between individual biomarkers and neuropsychological test scores. a Heatmap in training set. b Heatmap in testing set. See also legends to tables 1 and 3 for abbreviations.

Fig. 3. Variable importance plot for the domain of executive functioning. CTGF = Connective tissue growth factor. See also legend to table 1 for abbreviations.

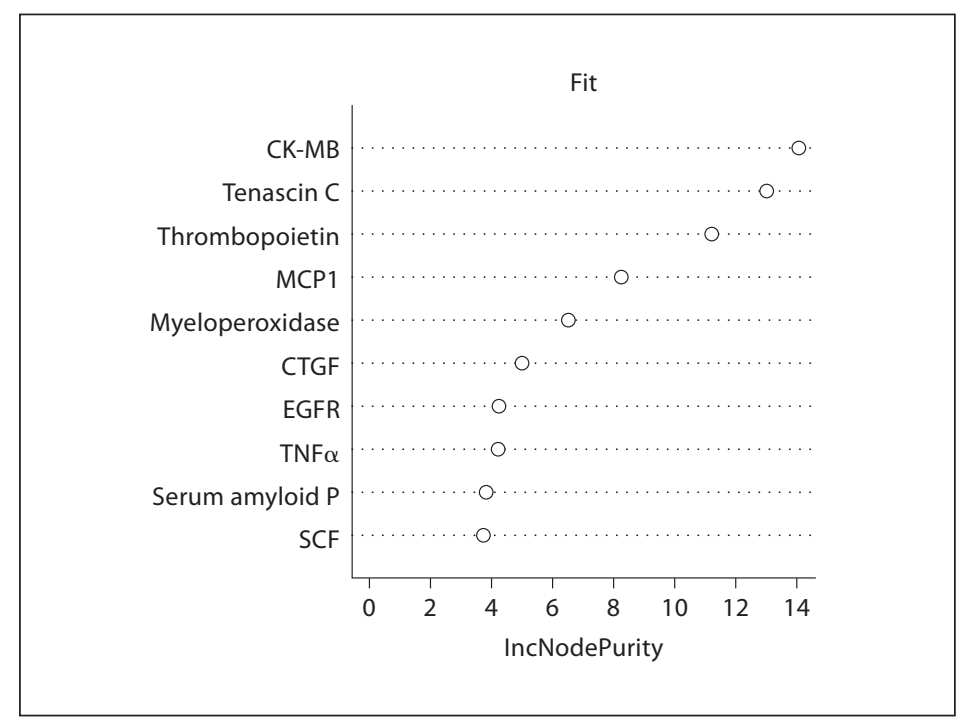


Fig. 4. Variable importance plot for the domain of memory. CA 19.9 = Cancer antigen 19.9; CEA = carcinoembryonic antigen. See also legend to table 1 for abbreviations.
O'Bryant et al.: Molecular Neuropsychology: Creation of Test-Specific Blood Biomarker Algorithms

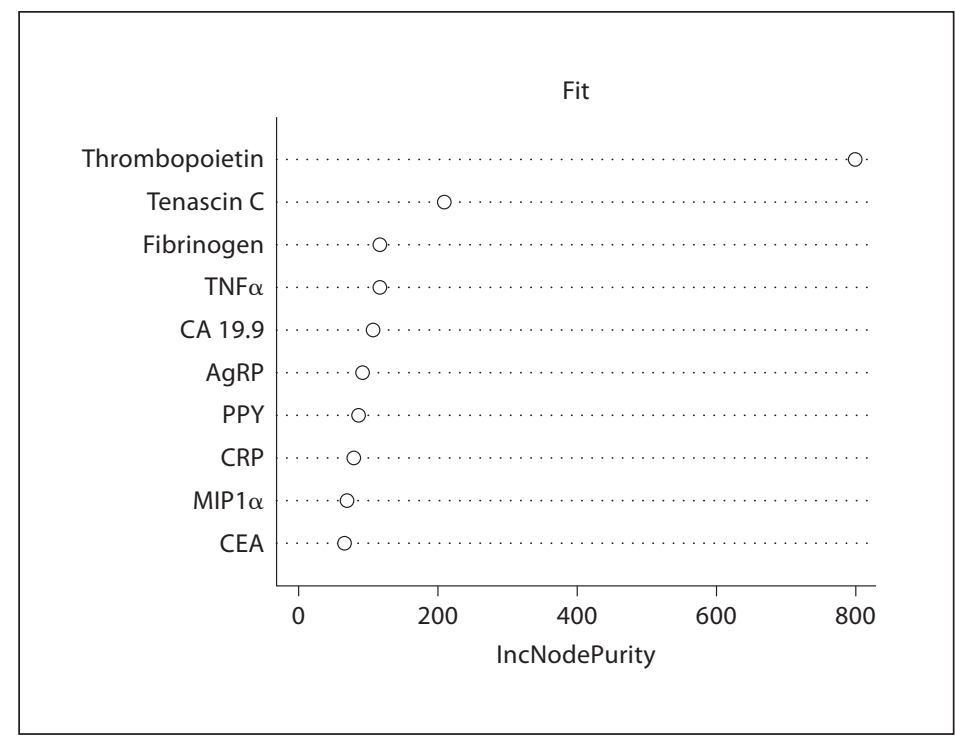

Table 3. Regression results predicting neuropsychological scores in the test set from the biomarker risk scores and biomarker algorithm created in the training set

\begin{tabular}{llllll}
\hline & B & SE & p value & \multicolumn{2}{l}{ Variance, \% } \\
\cline { 4 - 5 } & & & & $\begin{array}{l}\text { biomarker } \\
\text { risk score }\end{array}$ & $\begin{array}{l}\text { full biomarker } \\
\text { algorithm }\end{array}$ \\
\hline MMSE & & & & 28.7 & 37.6 \\
CDR SB & 1.29 & 0.15 & $<0.001$ & 33.5 & 42.3 \\
CDR GS & 1.53 & 0.15 & $<0.001$ & 34.3 & 41.6 \\
Clock Drawing Test & 1.51 & 0.15 & $<0.001$ & 24.6 & 32.0 \\
COWAT & 1.82 & 0.24 & $<0.001$ & 20.6 & 36.7 \\
BNT & 1.49 & 0.22 & $<0.001$ & 17.5 & 26.5 \\
AMNART & 1.38 & 0.22 & $<0.001$ & 6.6 & 37.0 \\
Trails A & 0.95 & 0.27 & $<0.001$ & 21.7 & 30.1 \\
Trails B & 1.80 & 0.26 & $<0.001$ & 12.9 & 22.4 \\
Digit span scale score & 1.67 & 0.27 & $<0.001$ & 43.9 & 49.4 \\
WMS LM immediate recall & 1.24 & 0.23 & $<0.001$ & 47.3 & 51.2 \\
WMS LM delayed recall & 1.86 & 0.17 & $<0.001$ & 30.9 & 35.3 \\
WMS VR immediate recall & 1.83 & 0.15 & $<0.001$ & 41.2 & 44.7 \\
WMS VR delayed recall & 1.59 & 0.18 & $<0.001$ & $<0.001$ & \\
\hline
\end{tabular}

$\mathrm{B}=$ Unstandardized $\beta ; \mathrm{SE}=$ standard error; variance - biomarker risk score $/$ full biomarker algorithm = variance in neuropsychological test scores accounted for by biomarker risk score alone/by biomarker risk score + demographics (age, gender, and education); CDR SB/GS = CDR scale sum of boxes score/global score; COWAT $=$ Controlled Oral Word Association Test scale score; Trails A/B = Trail Making Test Part A/B scale score.

test scores by test-specific biomarker algorithms (biomarker risk scores plus age, gender and education). The biomarker algorithms accounted for large portions of variance in neuropsychological test scores: including $49.4-51.2 \%$ of the variance in verbal memory, $33.5-44.7 \%$ of the variance in visual memory, $26.5-36.7 \%$ of the variance in language, $27.2-32.0 \%$ of the variance in executive functioning, and $30.1 \%$ of the variance in processing speed. We also 
Fig. 5. Variable importance plot for the domain of intelligence. CA 125 = Cancer antigen 125; MMP3 = matrix metallopeptidase 3. See also legend to table 1 for abbreviations.
Fig. 6. Variable importance plot for the domain of attention. MCP1 = Monocyte chemotactic protein-1; IgE = immunoglobulin E; MIP1 $\alpha=$ macrophage inflammatory protein 1 alpha. See legend to table 1 for abbreviations.
O'Bryant et al.: Molecular Neuropsychology: Creation of Test-Specific Blood Biomarker Algorithms
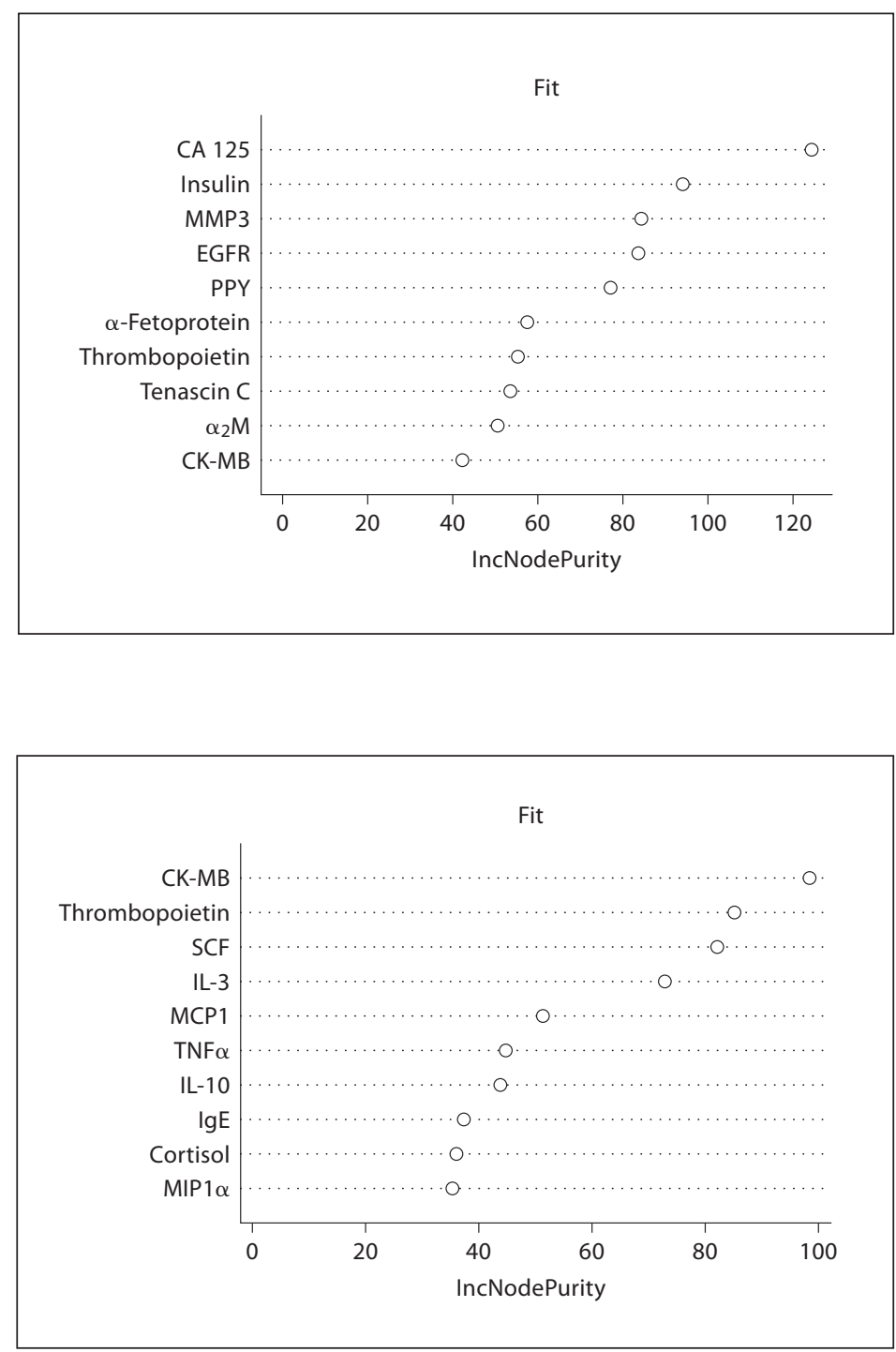

created a biomarker algorithm that accounted for $41.6-42.6 \%$ of the variance in disease severity (table 3 ). The amount of additional variance accounted for by including demographic factors varied by test and was smallest for memory measures.

Lastly, we reviewed the variable importance plots. RF generate variable importance plots based on the number of times each variable is selected by all individual trees in the ensemble (naive variable importance) [45]. The Gini importance method, utilized in the current study, incorporates a weighted mean of the improvement in the splitting criteria of the individual trees produced by each variable [45]. Review of the variable importance plots showed that the relative ranking of markers in the algorithm varied by every test-specific biomarker risk score in the analyses. To illustrate this, variable importance plots reflecting the top 10 markers contained in the algorithms of tests from four different domains (executive functioning, memory, intelligence, and attention) are presented in figures 3-6. It is worth noting that the Gini index relies on the assumptions of independence, and does not account for the dependency among the features. More advanced statistical methods may be needed to more accurately access the modeling fit. 
Dementia

\begin{tabular}{l|l}
\hline Dement Geriatr Cogn Disord 2014;37:45-57 \\
\hline DOI: $\underline{10.1159 / 000345605}$ & $\begin{array}{l}\text { (c) } 2013 \text { S. Karger AG, Basel } \\
\text { www.karger.com/dem }\end{array}$ \\
\hline
\end{tabular}

O'Bryant et al.: Molecular Neuropsychology: Creation of Test-Specific Blood Biomarker Algorithms

\section{Conclusion}

We took a novel approach to the search for biomarkers of cognitive functioning by shifting the outcome variable from dichotomous categorization of group status (case vs. control) to a continuous construct of neuropsychological test scores. Here we demonstrate that it is possible to create serum-based biomarker algorithms of specific neuropsychological functions that account for considerable portions of the variance in test scores and that the biomarker profiles vary according to cognitive domain. This proof-of-concept work supports expanding search for biomarker mediators of cognitive functioning, which we entitle molecular neuropsychology.

This work expands on prior work looking at how individual biomarkers are related to specific neuropsychological tests, which may have broader utility for understanding and predicting cognitive dysfunction. For example, it is possible that biomarker profiles will aid in the identification of those at greatest risk of cognitive decline. Prior work has demonstrated that baseline neuropsychological test scores predict status changes over time as well as progression to AD. For example, Musicco et al. [46] recently analyzed data on 154 newly diagnosed $\mathrm{AD}$ cases and found that more severe memory and executive functioning difficulties at baseline predicted more rapid progression over a 2-year follow-up period. Examining data from VITA (Vienna Transdanube Aging Study), Jungwirth et al. [47] analyzed 5-year longitudinal data to determine what baseline variables best predicted incident $A D$ in those without cognitive impairment. These authors found that a combination of the baseline CERAD Word List Delayed Recall, Trail Making Test Part A, presence of the APOE\&4 allele gene, and memory complaints significantly predicted incident $\mathrm{AD}$, with an area under the receiveroperating characteristic curve of 0.91 and a model $\mathrm{R}^{2}=0.43$. It is possible that combining biomarkers with select cognitive and clinical variables will significantly improve the prediction capacity of such models, and our group is currently working on this possibility. Another advantage of this approach is the possibility of studying biological systems that may be of etiological importance. Lastly, a significant amount of research has gone into imaging neurological mechanisms of cognitive function/dysfunction. The current work may provide the ability to combine biochemical pathway analysis with functional neuroimaging methods for a better understanding of the biology of neuropsychological dysfunction.

The current results also support the notion that our algorithms may be test specific based on the variable importance plots. In fact, the order of the markers varied across all neuropsychological tests (Clock Drawing Test, WMS LM I, AMNART, and Digit Span presented in fig. 1-4). While it has been suggested that the variable importance measures from RF analysis can be biased [45], the current findings provide ample proof of concept for delving further into the investigation of neuropsychological domain-specific blood-based biomarker algorithms. While we utilized a broad set of proteins, there are other proteins that have been found important that were not included in our multiplex assays [22]. It is certainly possible that better algorithms can be created by utilization of even larger protein panels that could then be refined to briefer versions.

There are limitations to the current study. While we utilized a broad range of serumbased proteins, it is likely that other markers not included in our assays would contribute significantly to building such blood-based biomarker algorithms of neuropsychological functioning. Future work should utilize a larger discovery set of biomarkers that can be narrowed as necessary. A second limitation to the current study is the brevity of the neuropsychological test battery. The TARCC battery consists of commonly used neuropsychological instruments; however, it was designed to be brief and does not fully assess several domains of cognition (e.g. executive functioning and visuospatial skills). In order to thoroughly research the potential of creating serum-based biomarker algorithms of neuropsychological tests and/or 
domains, a more comprehensive battery of tests should be implemented. A second limitation to the TARCC battery is the lack of consistency across sites with regard to instruments (e.g. WMS-R vs. WMS-III). We utilized scale scores in order to equate across test versions; however, this remains a limitation to the protocol. Our study is also limited by inclusion of only AD cases and normal controls. When examining only individuals who have been screened into research projects based on performing 'normal' or abnormal on cognitive measures creates an inherent bias that reduces the range of performance in neuropsychological test scores, as is the case with the TARCC cohort. Inclusion of AD cases in the current study enables us to capitalize on a full spectrum of cognitive performance. However, AD status was utilized in case selection thereby causing some unavoidable circularity. On the other hand, if one enters disease severity (or disease status) into the models, the results do not hold, which is likely due to the fact that disease severity and degree of neurocognitive deficits are highly confounded. If we analyzed the group separately, the current findings do not hold due to this restricted range of scores. The advantage of this approach is the ability to have a broad range of cognitive function/ dysfunction; however, additional work is needed to represent a full spectrum of neuropsychological status. For example, there is evidence to suggest that biomarkers will also be related to exceptional cognitive functioning, as demonstrated by Lopez et al. [48] who found that uromodulin and complement C3 were expressed at higher levels among highly intelligent elders when compared to those with lower intelligence.

The current study demonstrates that (1) blood-based biomarkers can be combined to create algorithms related to neuropsychological functioning, and (2) biomarker profiles will vary according to the cognitive domain being examined. This proof-of-concept work highlights the importance of investigating blood-based biomarker profiles of neurosychological functioning, which may have clinical utility across a broad range of conditions/populations.

\section{Acknowledgments}

Research reported in this publication was supported by the National Institute on Aging of the National Institutes of Health under Award Nos. R01AG039389 and P30AG12300. The content is solely the responsibility of the authors and does not necessarily represent the official views of the National Institutes of Health. This study was made possible by TARCC funded by the state of Texas through the Texas Council on Alzheimer's Disease and Related Disorders.

TARC investigators: Baylor College of Medicine: Rachelle Doody, Susan Rountree, Valory Pavlik, Eveleen Darby, Aline Hittle; Texas Tech University Health Science Center: Benjamin Williams, Andrew Dentino, Gregory Schrimsher, Parastoo Momeni, Ronnie Orozco; University of North Texas Health Science Center: Janice Knebl, Lisa Alvarez, Douglas Mains, Thomas Fairchild, Rosemary McCallum, Leigh Johnson, Hilda Benavides; University of Texas Southwestern Medical Center: Roger Rosenberg, Ryan Huebinger, Perrie Adams, Joan Reisch, Janet Smith, Mechelle Murray, Tomequa Sears; University of Texas Health Sciences Center - San Antonio: Donald Royal, Raymond Palmer.

\section{Disclosure Statement}

None. 
O'Bryant et al.: Molecular Neuropsychology: Creation of Test-Specific Blood Biomarker Algorithms

\section{References}

1 O’Bryant SE, Xiao G, Barber R, Reisch J, Doody R, Fairchild T, Adams P, Waring S, Diaz-Arrastia R: A serum protein-based algorithm for the detection of Alzheimer disease. Arch Neurol 2010;67:1077-1081.

- 2 Jack CR Jr, Knopman DS, Jagust WJ, Shaw LM, Aisen PS, Weiner MW, Petersen RC, Trojanowski JQ: Hypothetical model of dynamic biomarkers of the Alzheimer's pathological cascade. Lancet Neurol 2010;9:119-128.

3 Laske C, Fallgatter AJ, Stransky E, Hagen K, Berg D, Maetzler W: Decreased $\alpha$-synuclein serum levels in patients with Lewy body dementia compared to Alzheimer's disease patients and control subjects. Dement Geriatr Cogn Disord 2011;31:413-416.

4 O'Bryant SE, Hobson VL, Hall JR, Barber RC, Zhang S, Johnson L, Diaz-Arrastia R: Serum brain-derived neurotrophic factor levels are specifically associated with memory performance among Alzheimer's disease cases. Dement Geriatr Cogn Disord 2011;31:31-36.

5 Mayer AR, Mannell MV, Ling J, Gasparovic C, Yeo RA: Functional connectivity in mild traumatic brain injury. Hum Brain Mapp 2011;32:1825-1832.

6 Li J, Li XY, Feng DF, Pan DC: Biomarkers associated with diffuse traumatic axonal injury: exploring pathogenesis, early diagnosis, and prognosis. Trauma 2010;69:1610-1618.

- 7 Xiong P, Zeng Y, Wan J, XiaoHan DH, Tan D, Lu J, Xu F, Li Hy, Zhu Z, Ma M: The role of NGF and IL-2 serum level in assisting the diagnosis in first episode schizophrenia. Psychiatry Res 2011;189:72-76.

- 8 Levin Y, Wang L, Schwarz E, Koethe D, Leweke FM, Bahn S: Global proteomic profiling reveals altered proteomic signature in schizophrenia serum. Mol Psychiatry 2010;15:1088-1100.

- 9 Taurines R, Dudley E, Grassl J, Warnke A, Gerlach M, Coogan AN, Thome J: Review: proteomic research in psychiatry. J Psychopharmacol 2011;25:151-196.

10 Hall J, O’Bryant SE, Johnson LA, Barber RC: Depression and brain-derived neurotrophic factor levels in Alzheimer's disease. Neurosci Med 2011;2:43-47.

-11 Bocchio-Chiavetto L, Bagnardi V, Zanardini R, Molteni R, Gabriela Nielsen M, Placentino A, Giovannini C, Rillosi L, Ventriglia M, Riva MA, et al: Serum and plasma BDNF levels in major depression: a replication study and meta-analyses. World J Biol Psychiatry 2010;11:763-773.

12 O’Bryant SE, Xiao G, Barber R, Huebinger R, Wilhelmsen K, Edwards M, Graff-Radford N, Doody R, Diaz-Arrastia R, Texas Alzheimer's Research \& Care Consortium, Alzheimer's Disease Neuroimaging Initiative: A bloodbased screening tool for Alzheimer's disease that spans serum and plasma: findings from TARC and ADNI. PLoS ONE 2011;6:e28092.

13 O’Bryant S, Xiao G, Barber R, Reisch J, Hall J, Cullum CM, Doody R, Fairchild T, Adams P, Wilhelmsen K, DiazArrastia R: A blood based algorithm for the detection of Alzheimer's disease. Dement Geriatr Cogn Disord 2011;32:55-62.

14 Vemuri P, Gunter JL, Senjem ML, Whitwell JL, Kantarci K, Knopman DS, Boeve BF, Petersen RC, Jack CR Jr: Alzheimer's disease diagnosis in individual subjects using structural MR images: validation studies. Neuroimage 2008;39:1186-1197.

15 Zhang D, Wang Y, Zhou L, Yuan H, Shen D, Alzheimer's Disease Neuroimaging Initiative: Multimodal classification of Alzheimer's disease and mild cognitive impairment. Neuroimage 2011;55:856-867.

16 Brys M, Glodzik L, Mosconi L, Switalski R, De Santi S, Pirraglia E, Rich K, Kim BC, Mehta P, Zinkowski R, et al: Magnetic resonance imaging improves cerebrospinal fluid biomarkers in the early detection of Alzheimer's disease. J Alzheimers Dis 2009;16:351-362.

17 Benoit CE, Rowe WB, Menard C, Sarret P, Quirion R: Genomic and proteomic strategies to identify novel targets potentially involved in learning and memory. Trends Pharmacol Sci 2011;32:43-52.

18 Shaw LM, Korecka M, Clark CM, Lee VM, Trojanowski JQ: Biomarkers of neurodegeneration for diagnosis and monitoring therapeutics. Nat Rev Drug Discov 2007;6:295-303.

19 Schneider P, Hampel H, Buerger K: Biological marker candidates of Alzheimer's disease in blood, plasma, and serum. CNS Neurosci Ther 2009;15:358-374.

20 Folstein MF, Folstein SE, McHugh PR: 'Mini-Mental State'. A practical method for grading the cognitive state of patients for the clinician. J Psychiatr Res 1975;12:189-198.

-21 O’Bryant SE, Waring SC, Hobson V, Hall JR, Moore CB, Bottiglieri T, Massman P, Diaz-Arrastia R: Decreased C-reactive protein levels in Alzheimer disease. J Geriatr Psychiatry Neurol 2010;23:49-53.

-22 Wilson JS, Mruthinti S, Buccafusco JJ, Schade RF, Mitchell MB, Harrell DU, Gulati NK, Miller LS: Anti-RAGE and $A \beta$ immunoglobulin levels are related to dementia level and cognitive performance. J Geront A Biol Sci Med Sci 2009;64:264-271.

23 Randolph C: Repeatable Battery for the Assessment of Neuropsychological Status. San Antonio, Psychological Corporation, 1998.

24 Beers SR, Berger RP, Adelson PD: Neurocognitive outcome and serum biomarkers in inflicted versus noninflicted traumatic brain injury in young children. J Neurotrauma 2007;24:97-105.

25 Dik MG, Jonker C, Hack CE, Smit JH, Comijs HC, Eikelenboom P: Serum inflammatory proteins and cognitive decline in older persons. Neurology 2005;64:1371-1377.

26 Waring S, O’Bryant SE, Reisch JS, Diaz-Arrastia R, Knebl J, Doody R, Texas Alzheimer's Research Consortium: The Texas Alzheimer's Research Consortium longitudinal research cohort: study design and baseline characteristics. Texas Public Health J 2008;60:9-13. 
O'Bryant et al.: Molecular Neuropsychology: Creation of Test-Specific Blood Biomarker Algorithms

27 McKhann D, Drockman D, Folstein M, et al: Clinical diagnosis of Alzheimer's disease: report of the NINCDSADRDA Work Group. Neurology 1984;34:939-944.

28 Wechsler D: Wechsler Memory Scale, ed 3. San Antonio, Psychological Corporation, 1997.

29 Lezak MD, Howieson DB, Loring DW: Neuropsychological Assessment, ed 4. Oxford, Oxford University Press, 2004.

30 Strauss E, Sherman EMS, Spreen O: A Compendium of Neuropsychological Tests: Administration, Norms, and Commentary, ed 3. Oxford, Oxford University Press, 2006.

-31 Morris JC: Clinical dementia rating: a reliable and valid diagnostic and staging measure for dementia of the Alzheimer type. Int Psychogeriatr 1997;1:173-176.

-32 Ivnik R, Malec JF, Smith GE, Tangalos EG, Petersen RC, Kokmen E, Kurkland LT: Mayo's Older Americans Normative Studies: WAIS-R norms for age 56 to 97. Clin Neuropsychol 1992;6:1-30.

-33 Ivnik RJ, Malec JF, Smith GE, Tangalos EG, Petersen RC: Neuropsychological tests' norms above age 55 COWAT, BNT, MAE Token, WRAT-R Reading, AMNART, STROOP, TMT and JLO. Clin Neuropsychol 1996;10:262-278.

34 Psychological Corporation: WAIS-III - WMS-III Technical Manual. San Antonio, Psychological Corporation, 1997.

-35 Hobson V, Hall JR, Harvey M, Cullum CM, Lacritz L, Massman PJ, Waring SC, O’Bryant SE: An examination of the Boston Naming Test: calculation of 'estimated' 60-item score from 30- and 15-item scores in a cognitively impaired population. Int J Geriatr Psychiatry 2011;26:351-355.

36 R: a language and environment for statistical computing. http://www.R-project.org.

-37 Breiman L: Random forests. Machine Learning 2001;45:5-32.

38 Manual on setting up, using, and understanding random forests V3.1. http://oz.berkeley.edu/users/breiman/ Using_random_forests_V3.1.pdf.

-39 Wu B, Abbott T, Fishman D, McMurray W, Mor G, Stone K, Ward D, Williams K, Zhao H: Comparison of statistical methods for classification of ovarian cancer using a proteomics dataset. Bioinformatics 2003;19:16361643.

40 Díaz-Uriarte R, Alvarez de Andrés S: Gene selection and classification of microarray data using random forest. BMC Bioinformatics 2006; 7:3.

-41 Finehout EJ, Franck Z, Choe LH, Relkin N, Lee KH: Cerebrospinal fluid proteomic biomarkers for Alzheimer's disease. Ann Neurol 2007;61:120-129.

-42 Lehmann C, Koenig T, Jelic V, Prichep L, John RE, Wahlund LO, Dodge Y, Dierks T: Application and comparison of classification algorithms for recognition of Alzheimer's disease in electrical brain activity (EEG). J Neurosci Methods 2007;161:342-350.

43 Tripoliti EE, Fotiadis DI, Argyropoulou M: A supervised method to assist the diagnosis of Alzheimer's disease based on functional magnetic resonance imaging. Conf Proc IEEE Eng Med Biol Soc 2007:3426-3429.

44 Tripoliti EE, Fotiadis DI, Argyropoulou M: An automated supervised method for the diagnosis of Alzheimer's disease based on fMRI data using weighted voting schemes. IEEE 2008;2008:340-345.

45 Strobl C, Boulesteix AN, Zeileis A, Hothorn T: Bias in random forest variable importance measures: illustrations, sources and a solution. BMC Bioinformatics 2007;8:25.

46 Musicco M, Salamone G, Caltagirone C, Cravello L, Fadda L, Lupo F, Mosti S, Perri R, Palmer K: Neuropsychological predictors of rapidly progressing patients with Alzheimer's disease. Dement Geriatr Cogn Disord 2010; 30:219-228.

-47 Jungwirth S, Zehetmayer S, Bauer P, Weissgram S, Tragl KH, Fischer P: Prediction of Alzheimer dementia with short neuropsychological instruments. J Neural Transm 2009;116:1513-1521.

48 Lopez LM, Mullen W, Zürbig P, Harris SE, Gow AJ, Starr JM, Porteous DJ, Mischak H, Deary IJ: A pilot study of urinary peptides as biomarkers for intelligence in old age. Intelligence 2011;39:46-53. 